\title{
DILATIONS OF LIMIT ALGEBRAS AND INTERPOLATING SPECTRUM
}

\author{
Allan P. Donsig
}

We show that limit algebras having interpolating spectrum are characterized by the property that all locally contractive representations have $*$-dilations. This extends a result for digraph algebras by Davidson. It is an open question if such a limit algebra is the limit of a direct system of digraph algebras with interpolating digraphs, although a positive answer would allow one to obtain one direction of our result directly from Davidson's. Instead, we give a 'local' construction of digraph algebras with interpolating digraphs and use this to extend representations.

Tree algebras (in the sense of Davidson, Paulsen, and Power) have been characterized by a commutant lifting property among digraph algebras with interpolating digraphs. We show that the analogous result holds for limit algebras, i.e., limit algebras with the analogous spectral condition are characterized by the same commutant lifting property among the limit algebras with interpolating spectrum.

Dilation theory for operator algebras has its origin in the Sz.-Nagy dilation theorem, that every contractive operator on Hilbert space has a unitary dilation [SN]. That is, given a contractive operator $X$ on a Hilbert space $\mathcal{H}$, there a Hilbert space $\mathcal{K}$ containing $\mathcal{H}$ and a unitary operator $U$ on $\mathcal{K}$ so that $X^{n}=\left.P_{\mathcal{H}} U^{n}\right|_{\mathcal{H}}$ for $n \geq 1$. Ando's theorem [An] extends this result to two operators, i.e., any pair of commuting contractions has a unitary dilation. This fails for three or more operators $[\mathbf{P a}]$. Closely related to Ando's theorem is the Sz.-Nagy-Foiaş commutant lifting theorem [SNF1], that given a contraction $T$ with unitary dilation $U$ and a contraction $X$ commuting with $T$, there is a contractive dilation of $X$ commuting with $U$. For a unified treatment of these topics, see the monograph [SNF2].

From one point of view, these results are about dilating representations of the disk algebra. By von Neumann's inequality, each contraction $T$ induces a contractive representation of disk algebra, given by $f \mapsto f(T)$. Conversely, a contractive representation of disk algebra gives a contractive operator, the image of the function $f(z)=z$. Thus, Sz.-Nagy's theorem is equivalent to 
saying that every representation $\rho$ of the disk algebra that is locally contractive, in the sense that $\|\rho(f)\| \leq 1$ where $f$ is the function $f(z)=z$, can be dilated to a $*$-representation of the $\mathrm{C}^{*}$-algebra $\mathrm{C}(\mathbb{T})$.

The seminal papers of Arveson [A1, A2] consider the general problem: given a (nonselfadjoint) operator algebra, a subalgebra of a $\mathrm{C}^{*}$-algebra, and a representation of the operator algebra, when does the representation extend to a $*$-representation of the $\mathrm{C}^{*}$-algebra? The necessary and sufficient condition is that the original representation be completely contractive. To define this, suppose $\rho$ is the representation of the operator algebra $A$ contained in the $\mathrm{C}^{*}$-algebra $C$. Then there is a unique $\mathrm{C}^{*}$-norm on $C \otimes M_{n}$, as $M_{n}$ is finite dimensional, and this induces a norm on $A \otimes M_{n}$. Then $\rho$ is defined to be completely contractive if for all $n$, the maps $\rho \otimes 1_{M_{n}}$ are contractive on $A \otimes M_{n}$, with respect to this norm.

In [MM], McAsey and Muhly proved an analogue of Sz.-Nagy's theorem for analytic crossed products, which implies the theorem holds for finite dimensional nest algebras, i.e., contractive representations of the upper triangular matrix algebras admit dilations to the full matrix algebras. The paper $[\mathbf{P P W}]$ shows that locally contractive representations of finite dimensional nest algebras admit dilations and extends the result to $\sigma$-weakly continuous, contractive representations on infinite dimensional nest algebras, by using the semi-discreteness property. Ando's theorem and the Sz.-NagyFoiaş commutant lifting theorem are proved for nest algebras in [PP1] using similar methods. These results extend to CSL algebras under various conditions on the CSL algebra: [PPS] shows that for finite dimensional CSL algebras, a.k.a. digraph algebras, if the associated digraph is chordal, then all locally contractive representations are completely contractive and so admit dilations, and [DPP] shows that if the digraph is generated by a tree, then the digraph algebra satisfies an appropriate analogue of Sz. Nagy-Foiaş commutant lifting theorem. This last result extends to infinite dimensional completely distributive CSL algebras, provided the CSL algebra has properties analogous to those of digraph algebras generated by a tree. This extension is proved in much the same way as the extension argument of [PPW].

Recently, $[\mathbf{D}]$ has given necessary and sufficient conditions on the digraph for a digraph algebra to have every locally contractive representation completely contractive. Digraphs satisfying these conditions, called interpolating digraphs, include all chordal digraphs of course. Moreover, among all interpolating digraphs, those generated by trees are precisely the ones whose associated algebras satisfy the analogue of the commutant lifting theorem. Again, these results extend to suitable classes of completely distributive CSL algebras.

A different analogue of the commutant lifting theorem is considered 
in [MS4] and characterized, for digraph algebras, by a 'rooted' tree property. The papers [MS1-MS4] develop dilation theorems for various subalgebras of groupoid $\mathrm{C}^{*}$-algebras or of 'coordinatized' von Neumann algebras. This framework is quite general and one usually needs some condition on the subalgebra, such as triangularity, i.e., $A \cap A^{*}$ is maximal abelian in the generated $*$-algebra, or that $A+A^{*}$ is dense, in the appropriate topology, in the generated $*$-algebra.

Our focus is on limit algebras, algebras which are norm limits of digraph algebras. These algebras are contained in $\mathrm{AF} \mathrm{C}^{*}$-algebras and fit naturally into the framework of groupoid $\mathrm{C}^{*}$-algebras. The support of the operator algebra in the groupoid can be realized as a topological binary relation, a natural generalization of the digraph associated to digraph algebras. This topological relation, called the spectrum, is a complete invariant for triangular algebras, up to isometric isomorphism $[\mathbf{P 1}]$. The monograph $[\mathbf{P} 3]$ is a general reference for limit algebras.

In $[\mathbf{T}]$, Thelwall showed that a limit algebra with chordal spectrum can be written as a limit of digraph algebras with chordal digraphs. By applying the results of $[\mathbf{P P W}]$, it follows that every locally contractive representation of a limit algebra with chordal spectrum is completely contractive.

In this paper, we show that a limit algebra has interpolating spectrum if and only if every locally contractive representation is completely contractive. Unlike Thelwall's result, we do not show that a limit algebra with interpolating spectrum is the limit of a direct system of digraph algebras with interpolating digraph. Thus, we cannot directly apply the conclusions of [D]. Instead, we extend representations 'locally', adapting Thelwall's work to obtain digraph algebras with interpolating digraphs, and then using the structure of Davidson's 'sloppy unitary dilation'.

The second notable result is that, just as finite dimensional tree algebras are characterized by a commutant lifting property among digraph algebras with interpolating digraphs, limit algebras with the analogous spectral condition are characterized by the same property among limit algebras with interpolating spectrum. This result follows from combining the interpolating spectrum result, a theorem of [PP2] relating tensor products and the commutant lifting theorem, and a connection between interpolating digraphs and tensor products of tree algebras established in [D].

Much of this work was done at Lancaster University and I would like to thank the Department of Mathematics \& Statistics for its hospitality; I would like to thank Steve Power for helpful conversations and Ken Davidson for his comments on a preliminary version of this paper. 


\section{Limit Algebras and the Spectrum.}

In this section, we relate the properties of finite-dimensional subalgebras to properties of the limit algebra and of the spectrum. A digraph algebra, or finite dimensional CSL algebra, is a subalgebra of the $n \times n$ matrices over the complex numbers that contains the diagonal matrices. Given a digraph algebra $B$, we use $G(B)$ to denote associated digraph, i.e., the graph with vertices the diagonal matrix units $e_{i i}$ and an edge $\left(e_{i i}, e_{j j}\right)$ in the digraph if and only if the matrix unit $e_{j i}$ is in $B$.

Recall that a canonical masa $\mathcal{D}$ is a maximal abelian selfadjoint subalgebra (masa) of $\mathcal{C}$ so that there is a nested sequence of finite dimensional $\mathrm{C}^{*}$ algebras $\left(C_{i}\right)$ so that $\mathcal{C}=\overline{\cup_{i} C_{i}}$ and for all $i, D_{i}=C_{i} \cap \mathcal{D}$, is a masa in $C_{i}$ satisfying $\mathcal{D}=\overline{\cup_{i} D_{i}}$ and

$$
N_{D_{i}}\left(C_{i}\right) \subseteq N_{D_{i+1}}\left(C_{i+1}\right),
$$

where $N_{Y}(X)$ is

$\left\{x \in X: x\right.$ is a partial isometry and $x y x^{*}, x^{*} y x \in Y$ for all $\left.y \in Y\right\}$.

In general, we call $\mathcal{A} \subset \mathcal{C}$ a regular subalgebra if $D=\mathcal{A} \cap \mathcal{D}$ is a masa in $\mathcal{A}$ and $N_{D}(\mathcal{A}) \subseteq N_{\mathcal{D}}(\mathcal{C})$.

We assume that $\mathcal{A}$ is a subalgebra of an $\mathrm{AF} \mathrm{C}^{*}$-algebra $\mathcal{C}$ and that $\mathcal{A}$ contains a canonical masa $\mathcal{D}$. This implies that $\mathcal{A}$ is a regular subalgebra of $\mathcal{C}$ and also that the subalgebras $C_{i} \cap \mathcal{A}$ are digraph algebras and regular subalgebras of $\mathcal{A}$.

Henceforth, let $X$ be the maximal ideal space of $\mathcal{D}$ and for $p$ a projection in $\mathcal{D}$, let $\hat{p}$ denote $\{x \in X \mid x(p)=1\}$. Each $c \in N_{\mathcal{D}}(\mathcal{C})$ induces a partial homeomorphism $h_{c}$, from $\widehat{c c^{*}}$ to $\widehat{c^{*}} c$, where $h_{c}(x)$ is $d \mapsto x\left(c d c^{*}\right)$. If $\hat{c}$ denotes the graph of $h_{c}$ in $X \times X$, then we can define

$$
R(\mathcal{A})=\bigcup\left\{\hat{c}: c \in N_{\mathcal{D}}(\mathcal{A})\right\} .
$$

We topologize $R(\mathcal{A})$ by using as a basis of open sets $\hat{c}$ for $c \in N_{\mathcal{D}}(\mathcal{A})$. There are partially defined maps $\tilde{\alpha}_{i}: G\left(A_{i+1}\right) \rightarrow G\left(A_{i}\right)$, given by sending an edge $(a, b)$ to an edge $(c, d)$ if the matrix unit associated to $(a, b)$ appears in the sum of matrix units that is the image of the matrix unit associated to $(c, d)$. With these maps we can write $R(\mathcal{A})$ as an inverse limit, namely

$$
R(\mathcal{A})=\lim _{\longleftarrow}\left(G\left(A_{i}\right), \tilde{\alpha}_{i}\right) .
$$

We call $R(\mathcal{A})$ the spectrum of $\mathcal{A}$. It is a complete invariant for the pair $(\mathcal{A}, \mathcal{D})$, up to isometric isomorphism; see $[\mathbf{P} 1]$ or $[\mathbf{P} 3$, Chapter 7$]$. 
Suppose we write $\mathcal{A}$ as $\lim \left(A_{j}, \alpha_{j}\right)$ and choose matrix units systems for each $A_{j}$ so that matrix units in $A_{j}$ are sums of matrix units in $A_{j+1}$. Then we can describe the spectrum of $\mathcal{A}$ in terms of the matrix unit systems, which is convenient for computations with concrete examples; see $[\mathbf{H P}]$ or [P3, Chapter 7]. Specifically, $R(\mathcal{A})$ is the union of $\hat{e}$ as $e$ runs over the matrix units in all the $A_{j}$ and the topology has as a basis the set of $\hat{e}$ as $e$ runs over the matrix units

Definition 1.1. Suppose $Y$ is a finite subset of $X$. We call a finitedimensional regular subalgebra of $\mathcal{A}$, say $B$, a covering algebra for $\left.R(\mathcal{A})\right|_{Y \times Y}$ if

1. there is an isomorphism of digraphs $\phi:\left.G(B) \rightarrow R(\mathcal{A})\right|_{Y \times Y}$, and

2. we can identify $G(B)$ with matrix units of $B$ so that $\phi(e) \in \hat{e}$ for $e \in G(B)$.

Covering algebras are far from being unique. Indeed, suppose $B \subset \mathcal{A}$ is a covering algebra for $\left.R(\mathcal{A})\right|_{Y \times Y}, p \in B$ is a minimal diagonal projection and $y_{0} \in Y$ satisfies $\phi(p)=\left(y_{0}, y_{0}\right)$. For each clopen set $S$ with $y_{0} \in S \subset \hat{p}$, we can replace $p$ with the characteristic function of $S$ and similarly compress the other matrix units of $B$ to obtain a new covering algebra. In particular, if $Y \subset Z \subset X$, then it does not follow that a covering algebra for $\left.R(\mathcal{A})\right|_{Y \times Y}$ is a subalgebra of a covering algebra for $\left.R(\mathcal{A})\right|_{Z \times Z}$.

Lemma 1.2. If $Y$ is a finite subset of $X$, then we can find a covering algebra for $\left.R(\mathcal{A})\right|_{Y \times Y}$.

Conversely, if $B$ is a regular digraph subalgebra of $\mathcal{A}$, then there is a finite subset of $X$, call it $Y$, so that there is an injection from $G(B)$ to $\left.R(\mathcal{A})\right|_{Y \times Y}$.

Proof. Let $\mathcal{A}=\lim \left(A_{i}, \alpha_{i}\right)$. As usual, we identify the $A_{i}$ with subalgebras of $\mathcal{A}$ when convenient.

Since the topology in $R(\mathcal{A})$ separates points, we can find a $k$ so that for each diagonal matrix unit $e$ in $A_{k}$, $\hat{e}$ contains at most one point of $Y$. By increasing $k$, we can arrange that each point of $\left.R(\mathcal{A})\right|_{Y \times Y}$ is in the graph of some matrix unit in $A_{k}$. If we let $B \subset A_{k}$ be the span of the matrix units in $A_{k}$ that contain a point in $\left.R(\mathcal{A})\right|_{Y \times Y}$ then it is easy to check that $G(B)$ is isomorphic to $\left.R(\mathcal{A})\right|_{Y \times Y}$ and that the edge associated to a matrix unit in $B$ is sent to a point in the graph of that matrix unit. Being a span of normalizing matrix units, $B$ is a regular digraph subalgebra of $\mathcal{A}$.

Conversely, if $B$ is a regular digraph subalgebra of $\mathcal{A}$, then there is a system of normalizing matrix units $\left\{e_{i j}\right\}$ for $\mathrm{C}^{*}(B)$ so that $B$ is the span of the matrix units that it contains. Let $y \in R(\mathcal{A})$ be an element of $\hat{e}_{11}$ and let

$$
Y=\left\{h_{c}(y): c=e_{1 j} \text { for some } j \text { with } e_{j j} \in B\right\} .
$$


Clearly, there is a bijection between $Y$ and the set of minimal diagonal projections of $B$. It follows that there is an injection from $G(B)$ into $\left.R(\mathcal{A})\right|_{Y \times Y}$.

\section{Interpolating Digraphs and Algebras.}

After recalling the definition of interpolating digraphs and Davidson's characterization of digraph algebras with interpolating digraphs, we prove the main result, an analogous characterization for limit algebras with interpolating spectrum.

The $2 k$-cycle algebras and their digraphs, $D_{2 k}$, are fundamental examples, both for CSL algebras $[\mathbf{G H L}]$ and for limit algebras $[\mathbf{P 2}$, DoP]. The digraph $D_{2 k}$ has $2 k$ vertices, $\left\{u_{i}, v_{i} \mid 1 \leq i \leq k\right\}$, with edges from $u_{i}$ to $v_{i}$ and to $v_{i+1} \bmod k$, i.e.,

$$
A\left(D_{4}\right)=\left[\begin{array}{c|c}
* & * * \\
* & * * \\
\hline & * \\
& *
\end{array}\right]
$$

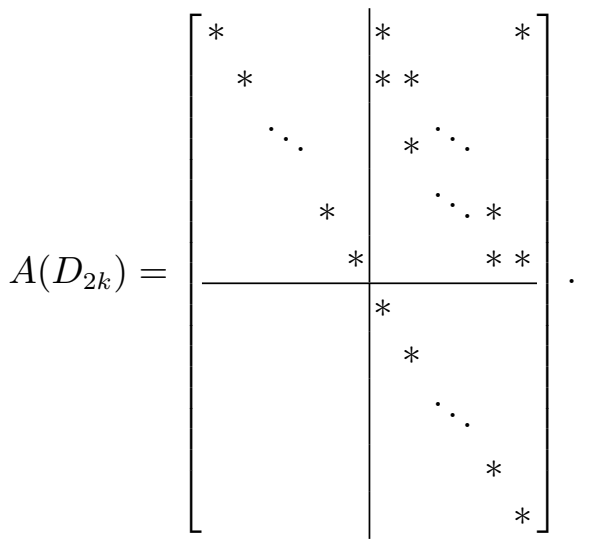

Let $\varphi_{k}: A\left(D_{2 k}\right) \rightarrow A\left(D_{2 k}\right)$ be the endomorphism which sends each matrix unit to itself, except the $(1, k+1)$ matrix unit, which is sent to its negative; these maps are locally contractive but not contractive, and so have no *dilation $[\mathbf{D}, \mathbf{D P}]$.

By a subgraph of a digraph $G$, we mean a subset of the vertices of $G$, together with all edges of $G$ between these vertices. Recall that a digraph is chordal if it does not contain a subgraph isomorphic to $D_{2 k}$ for $k \geq 2$, i.e., every cycle in the digraph has a chord. Also, if $G$ has a subgraph isomorphic to $D_{4}$, then call a vertex $e$ an interpolating vertex if every edge in the subgraph is a composition of edges through $e$, i.e., adding $e$ gives the 
digraph for the following algebra:

$$
\left[\begin{array}{r}
* * * \\
* * * * \\
e * * \\
* \\
\end{array}\right]
$$

Definition 2.1 [D]. A digraph $G$ is called interpolating if:

1. Every subgraph of $G$ isomorphic to $D_{4}$ has an interpolating vertex.

2. $G$ does not contain a subgraph isomorphic to $D_{2 k}$ for $k \geq 3$.

Clearly, all chordal digraphs are interpolating. The importance of interpolating digraphs is in the following theorem. For a digraph algebra $A$, a representation $\rho: A \rightarrow B(\mathcal{H})$ on a separable Hilbert space $\mathcal{H}$ is locally contractive if $\|\rho(e)\| \leq 1$ for all matrix units $e \in A$.

Theorem 2.2 [D, Corollary 3.5]. Let $A$ be a digraph algebra. The following are equivalent:

1. A has an interpolating digraph,

2. every locally contractive endomorphism of $A$ is contractive, and

3. every locally contractive representation has a unitary $*$-dilation.

If we think of the spectrum as the generalization of digraph for limit algebras, then our result is an extension of Davidson's.

Definition 2.3. Let $\mathcal{A}$ be a subalgebra of an $\mathrm{AF} \mathrm{C}^{*}$-algebra that contains a canonical masa. We say $R(\mathcal{A})$ is interpolating if for any finite subset $Y \subset X$, $\left.R(\mathcal{A})\right|_{Y \times Y}$ is never isomorphic to $D_{2 n}, n \geq 3$ and if it is isomorphic to $D_{4}$, then there is an interpolating vertex in $X$.

Together with Lemma 1.2, the next lemma shows that a limit algebra with interpolating spectrum contains many digraph algebras with interpolating digraphs.

Lemma 2.4. Let $\mathcal{A}$ be a subalgebra of an $A F \mathrm{C}^{*}$-algebra that contains a canonical masa. If $R(\mathcal{A})$ is interpolating and $Y \subset X$ is finite, then there is a finite set $Z$ with $Y \subset Z \subset X$ so that $\left.R(\mathcal{A})\right|_{Z \times Z}$ is interpolating.

Proof. Given that $R(\mathcal{A})$ is interpolating, the only way that $\left.R(\mathcal{A})\right|_{Y \times Y}$ can fail to be interpolating is if it contains some subgraph isomorphic to $D_{4}$ without containing an interpolating vertex. The following argument is adapted 
from [D, pp. 215-216]; the point is that we will add the needed interpolating vertices so that any new 4-cycles also have interpolating vertices.

Claim 1. If $B, C \subset Y$ with $(b, c) \in R(\mathcal{A})$ for all $b \in B$ and all $c \in C$, then there is $g \in Y$ with $(b, g),(g, c) \in R(\mathcal{A})$ for all $b \in B$ and all $c \in C$.

We prove the claim using induction on the sum of the cardinalities of $B$ and $C$. If $B$ or $C$ is a singleton, then we can set $g$ equal to that single element; if $B$ and $C$ both have cardinality two, then by the assumption on $R(\mathcal{A})$, a suitable $g$ exists. Suppose now $B=\left\{b_{1}, \ldots, b_{n}\right\}$ and $C=\left\{c_{1}, \ldots, c_{m}\right\}$. By induction, we can find an interpolating point $g_{1}$ for the sets $\left\{b_{1}, \ldots, b_{n-1}\right\}$ and $C$. Again by induction, there is an interpolating point $g$ for the sets $\left\{g_{1}, b_{n}\right\}$ and $C$. It is easy to check that $g$ has the required properties, proving the claim.

Let $\mathfrak{S}$ be the collection of maximal pairs of subsets of $Y,(B, C)$, with $(b, c) \in R(\mathcal{A})$ for all $b \in B, c \in C$. Clearly, $\mathfrak{S}$ is a finite collection, $\left\{S_{1}, \ldots, S_{m}\right\}$. Letting $S_{1}=\left(B_{1}, C_{1}\right)$, Claim 1 implies that there is a $g_{1} \in X$ so that $\left(b, g_{1}\right),\left(g_{1}, c\right) \in R(\mathcal{A})$ for all $b \in B_{1}, c \in C_{1}$. Next, we enlarge each $S_{i}=\left(B_{i}, C_{i}\right), i>1$, as follows. If $\left(g_{1}, c\right) \in R(\mathcal{A})$ for all $c \in C_{i}$, then we add $g_{1}$ to $B_{i}$. If $\left(b, g_{1}\right) \in R(\mathcal{A})$ for all $b \in B_{i}$, then we add $g_{1}$ to $C_{i}$. Otherwise, $S_{i}$ is left unchanged. Note that we may add $g_{1}$ to both $B_{i}$ and $C_{i}$ and that the new sets are maximal pairs of subsets of $Y \cup\left\{g_{1}\right\}$.

Continuing in this way for the (possibly modified) $S_{2}, \ldots, S_{m}$, we obtain $g_{2}, \ldots, g_{m}$, and we set $Z=Y \cup\left\{g_{1}, \ldots g_{m}\right\}$. Note that we do not try to add $g_{j}$ to $S_{i}$, for $i \leq j$.

Claim 2. $\left.R(\mathcal{A})\right|_{Z \times Z}$ is interpolating.

First, we show that $\left(g_{i}, g_{j}\right) \in R(\mathcal{A})$ if and only if the (original) sets satisfy $B_{i} \subset B_{j}$. By the maximality of $S_{i}$ and the choice of $g_{i}$, it follows that $B_{i}=\left\{b \in Y:\left(b, g_{i}\right) \in R(\mathcal{A})\right\}$. If $\left(g_{i}, g_{j}\right) \in R(\mathcal{A})$, then by transitivity

$$
B_{i}=\left\{b \in Y:\left(b, g_{i}\right) \in R(\mathcal{A})\right\} \subset\left\{b \in Y:\left(b, g_{j}\right) \in R(\mathcal{A})\right\}=B_{j} .
$$

Conversely, if $B_{i} \subset B_{j}$ then by maximality $C_{i} \supset C_{j}$ and so either $g_{j}$ has been added to $B_{i}$ or $g_{i}$ has been added to $C_{j}$. In either case, $\left(g_{i}, g_{j}\right) \in R(\mathcal{A})$.

To prove the claim, suppose that $\left.R(\mathcal{A})\right|_{T \times T}$ is a 4-cycle, i.e., $T=\left\{x_{1}, x_{2}\right.$, $\left.y_{1}, y_{2}\right\} \subseteq Z$ with $\left(x_{i}, y_{j}\right) \in R(\mathcal{A}), i, j \in\{1,2\}$. If $x_{i} \in Y$, let $E_{i}=\left\{x_{i}\right\}$ and if $x_{i}=g_{j}$ for some $j$, let $E_{i}=B_{j}$. If $y_{i} \in Y$, let $F_{i}=\left\{y_{i}\right\}$ and if $y_{i}=g_{j}$, let $F_{i}=C_{j}$. Then $\left(E_{1} \cup E_{2}, F_{1} \cup F_{2}\right)$ is contained in some maximal pair $\left(B_{k}, C_{k}\right)$ in $\mathfrak{S}$. It is straightforward to verify that $g_{k}$ is interpolating for $\left.R(\mathcal{A})\right|_{T \times T}$. 
This lemma does not imply that if a limit algebra has interpolating spectrum, then a finite-dimensional regular subalgebra is contained in such a subalgebra with interpolating spectrum. Indeed, this statement is equivalent to showing that every limit algebra with interpolating spectrum that has a presentation using digraph algebras with interpolating digraphs. However, if we work with finitely many algebras instead of only one, then the corresponding statement is true and this is enough to build the required representation.

Lemma 2.5. Suppose that $\mathcal{A}$ is a subalgebra of an $A F \mathrm{C}^{*}$-algebra containing a canonical masa $\mathcal{D}$, that $R(\mathcal{A})$ is interpolating, and that $S \subset \mathcal{A}$ is a finite-dimensional regular subalgebra. Then there are finite-dimensional regular subalgebras $B_{1}, \ldots, B_{m}$ so that each $G\left(B_{i}\right)$ is interpolating and the $\mathcal{D}$-bimodule generated by $B_{1}, \ldots, B_{m}$ contains $S$. Moreover, there are orthogonal projections $P_{1}, \ldots, P_{m}$ in the commutant of $S$ so that $P_{i} S \subseteq B_{i}$.

Proof. We suppose that $G(S)$ is connected; the general case then follows by considering the connected components of $G(S)$.

Fix a diagonal matrix unit in $S$, call it $e$. For each point $x \in \hat{e} \subseteq X$, let

$$
Y(x)=\{y \in X:(x, y) \text { or }(y, x) \in \hat{f} \text { for some matrix unit } f \in S\} .
$$

As $R(\mathcal{A})$ is interpolating, by Lemma 2.4 , there is a finite set $Z(x)$ containing $Y(x)$ so that $\left.R(\mathcal{A})\right|_{Z(x) \times Z(x)}$ is interpolating. By Lemma 1.2, there is a covering algebra, call it $A(e)$, for each $\left.R(\mathcal{A})\right|_{Z(x) \times Z(x)}$. Let $O(x)$ be the graph of the matrix unit in $A(x)$ that covers the point $(x, x) \in \hat{e}$. Then the set $\{O(x): x \in \hat{e}\}$ is an open cover of $\hat{e}$ and by the compactness of $\hat{e}$, it admits a finite subcover, given by $x_{1}, \ldots, x_{m}$, say. The bimodule generated by $A\left(x_{1}\right), \ldots, A\left(x_{m}\right)$ includes $e$ and all the other matrix units of $S$ and by construction each $A\left(x_{i}\right)$ has an interpolating digraph. Thus the $A\left(x_{i}\right)$ are almost the required subalgebras.

If the $O\left(x_{i}\right)$ overlap, then we can split the algebras $A\left(x_{i}\right)$ so the $O\left(x_{i}\right)$ are either disjoint or identical. Eliminating all but one algebra among those with identical sets $O\left(x_{i}\right)$ and changing $m$ if necessary, we have the required algebras $A\left(x_{1}\right), \ldots, A\left(x_{m}\right)$.

Let $Q_{i}$ be the projection in $B_{i}$ with graph $O\left(x_{i}\right)$. Let $P_{i}$ be the sum of $f^{*} Q_{i} f$ over all $f$ matrix units of $\mathrm{C}^{*}(S)$ with $f^{*} f=e$. Clearly, $P_{i}$ commutes with each matrix unit of $S$ and so is in the commutant of $S$. By construction, $P_{i} f \in A\left(x_{i}\right)$ for each matrix unit $f$ of $S$, so $P_{i} S \subseteq A\left(x_{i}\right)$, as required. Since the $O\left(x_{i}\right)$ are disjoint, it follows that the $P\left(x_{i}\right)$ are orthogonal.

If $\rho$ is a representation of a digraph algebra $A$, then $\rho$ being locally contractive is equivalent to having $\|\rho(e)\| \leq 1$ for all $e \in N_{D}(A)$ for some masa 
$D \subseteq A$. This motivates the following definition.

Definition 2.6. Let $\mathcal{A}$ be a subalgebra of an $\mathrm{AF} \mathrm{C}^{*}$-algebra so that $\mathcal{A}$ contains a canonical masa $\mathcal{D}$. A representation $\rho: \mathcal{A} \rightarrow B(\mathcal{H})$ on a separable Hilbert space $\mathcal{H}$ is locally contractive if, for all $e \in N_{\mathcal{D}}(\mathcal{A})$,

$$
\|\rho(e)\| \leq 1
$$

A major tool in the proof of Theorem 2.2 and an essential ingredient here is the vertex elimination scheme for anti-symmetric interpolating digraphs [ $\mathbf{D}$, Corollary 2.5]. That is, for an anti-symmetric interpolating digraph, there is a vertex so that deleting this vertex, and all edges involving this vertex, gives an interpolating sub-digraph. Moreover, this vertex has at most one immediate successor and at most one immediate predecessor. The result is that one can successively delete such vertices until one has the empty graph and so readily prove theorems by induction on the number of vertices.

We will also need the notion of a sloppy unitary dilation from [D], which is used there in proving Theorem 2.2. The sloppy unitary dilation of a contraction $T \in B(\mathcal{H})$ is a unitary $U \in B(\mathcal{K})$ where $\mathcal{K}=\mathcal{H}_{+} \oplus \mathcal{H} \oplus \mathcal{H}_{-}$and $\mathcal{H}_{-}, \mathcal{H}_{+}$are both countable direct sums of copies of $\mathcal{H}$ and where $U$ has the form

$$
U=\left[\begin{array}{ccc}
X & A & B \\
0 & T & C \\
0 & 0 & Y^{*}
\end{array}\right]
$$

with

1. $X, Y$ are isomorphic to $S \otimes I_{\mathcal{H}}$ where $S$ is the unilateral shift,

2. the range of $A$ has infinite codimension in $\left(I-X X^{*}\right) \mathcal{H}_{+}$, and

3. the domain of $C$ has infinite codimension in $\left(I-Y Y^{*}\right) \mathcal{H}_{-}$.

If $V$ is another sloppy unitary dilation of $T$, then there is a unitary $W=$ $W_{+} \oplus I \oplus W_{-}$with $W V W^{*}=U[\mathbf{D}$, Lemma 3.1].

Theorem 2.7. $\quad$ Let $\mathcal{A}$ be a unital subalgebra of an $A F \mathrm{C}^{*}$-algebra that contains a canonical masa $\mathcal{D}$. Then the spectrum, $R(\mathcal{A})$, is interpolating, if and only if, every locally contractive representation of $\mathcal{A}$ has a unitary *-dilation.

Proof. Let $X$ be the maximal ideal space of $\mathcal{D}$; since $\mathcal{A}$ is unital, $X$ is compact.

$(\Longrightarrow) \quad$ Fix a presentation for $\mathcal{A}$, i.e., $\mathcal{A}=\lim \left(A_{i}, \alpha_{i}\right)$. 
Suppose $\rho: \mathcal{A} \rightarrow B(\mathcal{H})$ is a locally contractive representation. If $\rho$ is not completely contractive, then a straightforward argument using the density of $\cup A_{i}$ in $\mathcal{A}$ shows that there is some $n$ so that $\left.\rho\right|_{A_{n}}$ is not completely contractive. Thus, it suffices to show that for each $n,\left.\rho\right|_{A_{n}}$ has a unitary *-dilation.

Fix $n$. By Lemma 2.5, we have regular subalgebras $B_{1}, \ldots, B_{m}$, with each $G\left(B_{i}\right)$ interpolating and $A_{n}$ contained in the bimodule generated by $B_{1}, \ldots, B_{m}$. There is some $k$ so that $B_{i} \subseteq A_{k}$ for all $i$. By possibly splitting each $B_{i}$ into several isomorphic algebras, we may assume that the matrix units of each $B_{i}$ are matrix units of $A_{k}$.

Next, we put $\left.\rho\right|_{A_{k}}$ into a standard form. Let $\left\{e_{i j}\right\}$ be the matrix units of $\mathrm{C}^{*}\left(A_{k}\right)$. We may assume that each $\rho\left(e_{i i}\right)$ is a projection of infinite rank by adding, if necessary, infinitely many copies of the identity representation. Thus we can identify $\rho\left(e_{i i}\right) \mathcal{H}$ for all $i$, call it $\mathcal{H}^{\prime}$, and so may regard $\left.\rho\right|_{A_{k}}$ as a representation on $M_{l}\left(B\left(\mathcal{H}^{\prime}\right)\right)$ for some $l$.

As $\rho$ is locally contractive, each $\rho\left(e_{i j}\right)$ is a contraction from the $j$-th copy of $\mathcal{H}^{\prime}$ to $i$-th copy. By Theorem 2.2, each $\left.\rho\right|_{B_{i}}$ can be dilated to a *-representation of $\mathrm{C}^{*}\left(B_{i}\right)$, call it $\sigma_{i}$. Moreover, these dilations have a common form. Let $\mathcal{K}^{\prime}$ be the $\ell^{2}$ direct sum of countably many copies of $\mathcal{H}^{\prime}$ and identify $\mathcal{H}^{\prime}$ with one of these copies, so that $\mathcal{H}^{\prime} \subset \mathcal{K}^{\prime}$. Then we may assume that the all $*$-dilations have a common range, namely $M_{l}\left(B\left(\mathcal{K}^{\prime}\right)\right)$. Each matrix unit of $\mathrm{C}^{*}\left(A_{k}\right)$ is in at most one of $\mathrm{C}^{*}\left(B_{1}\right), \ldots, \mathrm{C}^{*}\left(B_{m}\right)$, and if $e_{i j}$ is in one of these algebras, then its image is a unitary sending the $j$-th copy of $\mathcal{K}^{\prime}$ to the $i$-th copy.

Recall, by Lemma 2.5 that we have orthogonal projections $P_{1}, \ldots, P_{m}$ in the commutant of $A_{n}$ with $P_{i} A_{n} \subseteq B_{i}$ for each $i$. Since $P_{i}$ are pairwise orthogonal, so are their images, the $\rho\left(P_{i}\right)$. Then we can define $\sigma: A_{n} \rightarrow$ $M_{l}\left(B\left(\mathcal{K}^{\prime}\right)\right)$ by

$$
\sigma(a)=\sum_{i=1}^{m} \rho\left(P_{i}\right) \sigma_{i}\left(P_{i} a\right) \rho\left(P_{i}\right) .
$$

The map $\sigma$ is contractive, as an element $a$ is the direct sum of the $P_{i} a$ as $i$ runs from 1 to $m$ and the $\rho\left(P_{i}\right)$ are pairwise orthogonal.

Finally, $\sigma$ extends to a $*$-representation on $\mathrm{C}^{*}(A)$, if and only if, the partial isometries $\sigma(e), e$ a matrix unit, satisfy the same relations as the corresponding matrix units. As each $\sigma_{i}$ extends to a $*$-representation, the $\sigma_{i}\left(P_{i} e\right)$ do satisfy the required relations. As the $\rho\left(P_{i}\right)$ are pairwise orthogonal, each $\sigma(e)$ is the direct sum of the the $\sigma_{i}\left(P_{i} e\right)$ and so also satisfies these relations.

$(\Longleftarrow)$ We suppose that $R(\mathcal{A})$ is not interpolating and construct a locally contractive representation of $\mathcal{A}$ that is not contractive and hence has no *dilation; this argument is an elaboration on one direction of [ $\mathbf{D}$, Theorem 2.2]. 
By our supposition, there is a finite subset of $X$, say $Y$, so that $\left.R(\mathcal{A})\right|_{Y \times Y}$ is a 4-cycle without an interpolating vertex in $R(\mathcal{A})$, or is a $2 k$-cycle, $k \geq 3$. Suppose $Y$ is $\left\{u_{1}, \ldots, u_{k}, v_{1}, \ldots v_{k}\right\}, k \geq 2$, where $\left(u_{i}, v_{i}\right)$ and $\left(u_{i}, v_{i+1}\right)$ are in $R(\mathcal{A})$ (and $v_{k+1}=v_{1}$ ). For each $l$, define $U_{i}$ for $i=1, \ldots, k$ by

$$
U_{i}=\left\{y \in X \mid\left(u_{i}, y\right),\left(y, u_{i}\right) \in R(\mathcal{A})\right\},
$$

and $V_{j}$ similarly. For $i, j=1, \ldots, k$, let

$$
I_{i j}=\left\{y \in X \mid\left(u_{i}, y\right),\left(y, v_{j}\right) \in R(\mathcal{A}) \text { and } y \notin U_{i}, V_{i}\right\} .
$$

Finally, we set $H=\left\{y \in X \mid\left(u_{i}, y\right),\left(y, v_{j}\right)\right.$ for some $\left.i, j\right\}$, i.e., the union of the $U_{i}$, the $V_{j}$ and the $I_{i j}$.

Note that $H$ is countable, so we can introduce a separable Hilbert space $\mathcal{H}$ with orthonormal basis $\left\{b_{h}: h \in H\right\}$. We define a map $\phi:\left.R(\mathcal{A})\right|_{H \times H} \rightarrow$ $B(\mathcal{H})$ given by

$$
\phi(x, y)=\alpha(x, y) b_{x} b_{y}^{*},
$$

where $b_{x} b_{y}^{*}$ is the rank one operator $z \mapsto\left(z, b_{y}\right) b_{x}$ and $\alpha$ is given by

$$
\alpha(a, b)=\left\{\begin{aligned}
-1 & \text { if }(a, b) \in\left(U_{1} \cup\left(I_{11} \cap I_{1 k}\right)\right) \times\left(V_{1} \cup\left(I_{11} \backslash I_{1 k}\right)\right), \\
1 & \text { otherwise }
\end{aligned}\right.
$$

It is straightforward to check that $\alpha(a, b) \alpha(b, c)=\alpha(a, c)$ and hence $\phi(a, b) \phi(b, c)=\phi(a, c)$. Remark 5.6 below motivates this definition. If $H=Y$, then $\phi$ is $\varphi_{k}$, the locally contractive but not completely contractive representation defined at the start of this section. We can extend $\phi$ to $R(\mathcal{A})$ by sending elements outside $\left.R(\mathcal{A})\right|_{H \times H}$ to zero.

To define a representation $\Phi: \mathcal{A} \rightarrow B(\mathcal{H})$, we first define $\Phi$ on $N_{\mathcal{D}}(\mathcal{A})$ by sending such an element $e$ to

$$
\begin{aligned}
\Phi(e)= & \text { WOT }-\sum \phi(x, y) . \\
& (x, y) \in \hat{e}
\end{aligned}
$$

As each $\phi(x, y)$ has orthogonal initial and final projections, this sum does indeed converge in the weak operator topology. Being a partial isometry, $\Phi(e)$ has norm at most 1.

Extend $\Phi$ by linearity to linear combinations of elements of $N_{\mathcal{D}}(\mathcal{A})$. It is not hard to check that this map is bounded and so we may extend by continuity to obtain a locally contractive representation.

It remains only to show that $\Phi$ is not contractive. By Lemma 1.2, we can find a covering algebra for $\left.R(\mathcal{A})\right|_{Y \times Y}$, say $B$, with $G(B)=D_{2 k}$. As the graph of each matrix unit contains a point of $\left.R(\mathcal{A})\right|_{Y \times Y}$, it follows that 
compressing $\left.\Phi\right|_{B}$ by $P=\sum_{y \in Y} b_{y} b_{y}^{*}$ gives a representation isomorphic to $\varphi_{2 k}$. Hence $\left.\Phi\right|_{B}$ is not contractive.

Remark 2.8. An example is the best motivation for the definition of the maps $\alpha$ and $\phi$ in the previous proof. Suppose that $k=2$ and $A_{1}$ is the following matrix algebra

$$
\left[\begin{array}{lllll}
u_{1} & * & * & * & * \\
& u_{2} & * & * & * \\
& * & * & \\
& & * & * & * \\
& & & v_{1} & \\
& & & & v_{2}
\end{array}\right]
$$

where we suppose that $Y=\left\{u_{1}, u_{2}, v_{1}, v_{2}\right\}$. To obtain an extension of $\varphi_{2}$, the $(1,5)$ matrix unit must go to its negative. As the map is multiplicative, for each of the pairs of matrix units, $(1,3),(3,5)$ and $(1,4),(4,5)$, one of the pair must go to its negative. We send the $(1,3)$ and $(4,5)$ matrix units to their negatives, corresponding to the sets $U_{1} \times\left(I_{11} \backslash I_{1 k}\right)$ and $\left(I_{11} \cap I_{1 k}\right) \times V_{1}$ in the definition of $\alpha$.

Example 2.9. Another way one might prove the first direction of Theorem 2.7 is to first show that an interpolating spectrum implies the algebra has a presentation using digraph algebras with interpolating digraphs. It would then be straightforward to apply the characterization for digraph algebras to obtain the result. Indeed, replacing interpolating with chordal, this is the method that Thelwall uses in showing chordal spectrum implies contractive representations are completely contractive.

However, it is unclear how to construct the required presentation. The essential step is, given a limit algebra $\mathcal{A}$ with interpolating spectrum and a finite dimensional regular subalgebra $A_{1}$ to construct another finite dimensional regular subalgebra $A_{2}$ with $A_{1} \subset A_{2}$ and $G\left(A_{2}\right)$ interpolating. Lemma 2.5 is insufficient, as the algebra generated by the algebras $B_{1}, \ldots$, $B_{m}$ of the lemma need not be interpolating.

Concretely, consider

$$
A_{1}=\left[\begin{array}{cc|cc}
* & * & * & * \\
* & * & * \\
* & * & * \\
\hline & * & \\
& * & *
\end{array}\right], \quad \text { and } \quad A_{2}=\left[\begin{array}{ccc}
D & S_{12} & S_{13} \\
0 & S_{22} & S_{23} \\
0 & 0 & D
\end{array}\right]
$$

where each $D$ is the $12 \times 12$ diagonal matrices, $S_{22}$ is a $4 \times 4$ matrix and the two-by-two block of $S_{i j}$ 's is given by the $*$-diagram in the following matrix. 
The inclusion from $A_{1}$ to $A_{2}$ is given by sending the $(1,2)$ block of $A_{1}$ to the $(1,3)$ block of $A_{2}$, precisely, to the numbered images in the matrix. Then the $(1,1)$ and $(2,2)$ blocks of $A_{1}$ are sent to the $(1,1)$ and $(3,3)$ blocks of $A_{2}$, so that the resulting map from $A_{1}$ to $A_{2}$ is a homomorphism, albeit non-unital.

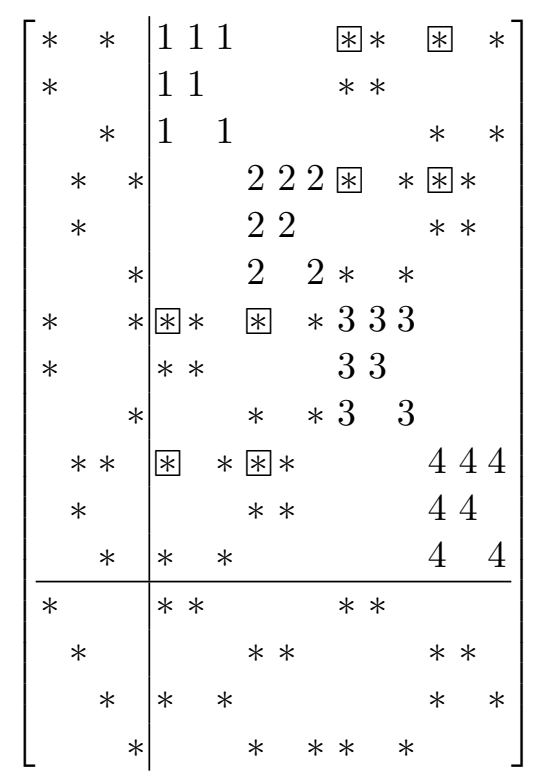

Observe that each of the 4-cycle of partial isometries in $A_{1}$,

$$
e_{1,4}, e_{1,5}, e_{2,5}, e_{2,4} \quad \text { and } \quad e_{1,4}, e_{1,6}, e_{3,6}, e_{3,4}
$$

is a sum of four 4-cycles in $A_{2}$ and each of these 4-cycles in $A_{2}$ has an interpolating point. However, the algebra generated by these 4-cycles and their interpolating points includes new 4-cycles that do not have interpolating points, namely the two boxed 4-cycles in the diagram. What one needs is some new construction that either does not introduce these new 4-cycles, or more likely, also constructs interpolating points for them.

\section{Tree Algebras.}

We characterize, among the limit algebras with interpolating spectrum, those which have a commutant lifting property or satisfy Ando's theorem. We say an algebra has the commutant lifting property if given a contractive representation $\rho$ and a contraction $X$ commuting with the range of $\rho$, there is a $*$-dilation $\pi$ and a unitary $U$ commuting with the range of $\pi$ so that $X=\left.P_{\mathcal{H}} U\right|_{\mathcal{H}}$. An algebra satisfies Ando's theorem if given a contractive 
representation of the algebra and a commuting representation of the disk algebra, then there are commuting $*$-dilations; this is equivalent to a stronger commutant lifting property, where $U$ is required to be a power dilation of $X$.

Recall that if $A$ is a digraph algebra, then the reduced digraph associated to a digraph algebra $A$, denoted $G_{r}(A)$, is $G(A) / \approx$ where $p_{i} \approx p_{j}$ if $\left(p_{i}, p_{j}\right)$ and $\left(p_{j}, p_{i}\right)$ are edges of $G(A)$. This digraph, $G_{r}(A)$, is a partial order on $\left\{p_{1}, \ldots, p_{n}\right\} / \approx$. The advantage of the reduced digraph is that its vertices correspond to the summands of $A \cap A^{*}$. Thus, $G_{r}(A)$ gives the non-selfadjoint structure of $A$ whereas $G(A)$ is also encumbered with information about $A \cap A^{*}$.

Definition 3.1. A digraph algebra $A$ is a tree algebra if each connected component of $G_{r}(A)$ is generated by a bilateral tree, a bilateral tree being an undirected finite tree in the usual sense with arbitrary directions specified for each edge.

In $[\mathbf{D P P}]$, tree algebras are shown to possess the commutant lifting property and to satisfy Ando's theorem. Further, in $[\mathbf{D}]$ it is shown that, for $A$ a digraph algebra with interpolating digraph, $A$ is a tree algebra if and only if it has the commutant lifting property.

The next theorem gives a local characterization of tree algebras, in terms of the associated digraph. In particular, tree algebras have interpolating digraphs.

Theorem 3.2. [DPP] For $A$ a digraph algebra, $A$ is a tree algebra if and only if $G(A)$ satisfies the following conditions:

1. If a subgraph of $G(A)$ contains the following digraph, then it also contains an edge between $f_{1}$ and $f_{2}$.

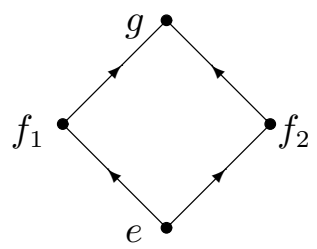

2. Every subgraph of $G(A)$ isomorphic to $D_{4}$ has an interpolating vertex, and

3. $G(A)$ does not contain a subgraph isomorphic to $D_{2 k}$ for $k \geq 3$.

Since the three conditions above are all local, we can extend them to the spectrum of a limit algebra in the same way that we defined interpolating 
spectrum.

Definition 3.3. $\quad$ Let $\mathcal{A}$ be a subalgebra of an $\mathrm{AF} \mathrm{C}^{*}$-algebra that contains a canonical masa. We say $R(\mathcal{A})$ is locally generated by trees if for any finite subset $Y \subset X$, the directed graph $\left.R(\mathcal{A})\right|_{Y \times Y}$ satisfies the three conditions of Theorem 3.2.

Since $R(\mathcal{A})$ is infinite, the conditions of Theorem 3.2 do not imply that each connected component of $R(\mathcal{A})$ is generated by a single tree; hence our somewhat awkward term for a spectrum satisfying these conditions.

Recall from [PP2] the minimal operator norm on a tensor product of two operator algebras. Given completely isometric isomorphisms $\tau_{i}: \mathcal{A}_{i} \rightarrow B\left(\mathcal{H}_{i}\right)$ for $i=1,2$, we have a norm on the algebraic tensor product of $\mathcal{A}_{1}$ and $\mathcal{A}_{2}$, defined by

$$
\left\|a_{1} \otimes a_{2}\right\|=\left\|\tau_{1}\left(a_{1}\right) \otimes \tau_{2}\left(a_{2}\right)\right\|_{B\left(\mathcal{H}_{1} \otimes \mathcal{H}_{2}\right)} .
$$

By [PP2, Theorem 2.2], this norm is independent of the choice of $\tau_{1}$ and $\tau_{2}$ and equals the spatial operator norm. In general this norm is not minimal among the complete operator cross-norms [ER]. Since we will have no cause to refer to other tensor product norms, here $\mathcal{A}_{1} \otimes \mathcal{A}_{2}$ will always refer to the completion of the algebraic tensor product with respect to this norm.

Our interest in this operator norm is motivated by the following pair of results. Given algebras $\mathcal{A}_{1}$ and $\mathcal{A}_{2}$, and representations $\tau_{i}: \mathcal{A}_{i} \rightarrow B(\mathcal{H})$ so that $\tau_{1}\left(a_{1}\right) \tau_{2}\left(a_{2}\right)=\tau_{2}\left(a_{2}\right) \tau_{1}\left(a_{1}\right)$ for all $a_{1} \in \mathcal{A}_{1}$ and $a_{2} \in \mathcal{A}_{2}$, we use $\tau_{1} \odot \tau_{2}$ to denote the representation of $\mathcal{A}_{1} \otimes \mathcal{A}_{2}$ on $\mathcal{H}$ given by

$$
\left(\tau_{1} \odot \tau_{2}\right)\left(a_{1} \otimes a_{2}\right)=\tau_{1}\left(a_{1}\right) \otimes \tau_{2}\left(a_{2}\right) .
$$

A unital operator algebra $\mathcal{A}$ has the commutant lifting property if and only if for all pairs of commuting completely contractive representations, $\tau_{1}$ of $\mathcal{A}$ and $\tau_{2}$ of $T_{2}$, the representation $\tau_{1} \odot \tau_{2}$ of $\mathcal{A} \otimes T_{2}$ has a $*$-dilation [PP2, Propositions 2.5 and 2.6]. An operator algebra $\mathcal{A}$ satisfies Ando's theorem if and only if for all $n$ and for all pairs of commuting completely contractive representations, $\tau_{1}$ of $\mathcal{A}$ and $\tau_{2}$ of $T_{n}$, the representation $\tau_{1} \odot \tau_{2}$ of $\mathcal{A} \otimes T_{n}$ has a $*$-dilation [PP2, Theorem 3.2].

If $\mathcal{A}_{i}, \mathcal{B}_{i}$ are operator algebras and $\mathcal{A}_{i}$ is a subalgebra of $\mathcal{B}_{i}$ for $i=1,2$, then the natural inclusion of $\mathcal{A}_{1} \otimes \mathcal{A}_{2}$ into $\mathcal{B}_{1} \otimes \mathcal{B}_{2}$ is a complete isometry [PP2, Corollary 2.3]. In particular, if $\mathcal{A}=\underline{\lim }\left(A_{i}, \alpha_{i}\right)$ then as the $\alpha_{i}$ are *-extendible, they are complete isometries and $\overrightarrow{\mathrm{so}}$ the injection

$$
A_{i} \otimes T_{n} \stackrel{\alpha_{i} \otimes \mathrm{Id}}{\longrightarrow} A_{i+1} \otimes T_{n}
$$

is a complete isometry (and hence $*$-extendible). This map is easily seen to be regular and so we have a limit algebra $\lim \left(A_{i} \otimes T_{n}, \alpha_{i} \otimes \mathrm{Id}\right)$, which is isomorphic to $\mathcal{A} \otimes T_{n}$. 
With these results, we can now prove the main theorem of this section. The arguments are adaptations of those in [D, Section 4].

Theorem 3.4. Let $\mathcal{A}$ be a unital subalgebra of an $A F \mathrm{C}^{*}$-algebra that contains a canonical masa $\mathcal{D}$ and suppose that $R(\mathcal{A})$ is interpolating. The following are equivalent:

1. The spectrum, $R(\mathcal{A})$, is locally generated by trees,

2. $\mathcal{A}$ satisfies Ando's theorem, and

3. $\mathcal{A}$ has the commutant lifting property.

Proof. $(1 \Longrightarrow 2) \quad$ To show $\mathcal{A}$ satisfies Ando's theorem, it suffices to show that for every $n$ we can lift a representation $\tau_{1} \odot \tau_{2}$ of $\mathcal{A} \otimes T_{n}$. By the previous discussion,

$$
\mathcal{A} \otimes T_{n}=\underset{\lim }{\longrightarrow}\left(A_{i} \otimes T_{n}, \alpha_{i} \otimes \mathrm{Id}\right),
$$

and so $R\left(\mathcal{A} \otimes T_{n}\right)=R(\mathcal{A}) \otimes G\left(T_{n}\right)$. Lemma 4.4 and Theorem 4.6 of [D] show that a digraph is a tree digraph if and only if its tensor product with each $G\left(T_{n}\right)$ is interpolating. It follows that $R\left(\mathcal{A} \otimes T_{n}\right)$ is interpolating if and only if $R(\mathcal{A})$ is locally generated by trees. By Theorem 2.7, each representation of $\mathcal{A} \otimes T_{n}$, and in particular, each representation of the form $\tau_{1} \odot \tau_{2}$, has a *-dilation.

$(2 \Longrightarrow 3)$ This is immediate from the formulation above of both conditions in terms of tensor products.

$(3 \Longrightarrow 1) \quad$ Suppose the spectrum is not locally generated by trees.

In the proof of $[\mathbf{D}$, Theorem 4.6], Davidson shows that for a digraph algebra $A$ with interpolating digraph, if $A$ is not a tree algebra, then the digraph of $A \otimes T_{2}$ is not interpolating. It follows that if the spectrum of $\mathcal{A}$ is not locally generated by trees, then the spectrum of $\mathcal{A} \otimes T_{2}$ is not interpolating.

Hence by Theorem $2.7, \mathcal{A} \otimes T_{2}$ has a locally contractive representation, say $\tau$, that does not have a $*$-dilation. Let $\lambda_{1}$ be the inclusion of $\mathcal{A}$ in $\mathcal{A} \otimes T_{2}$ and $\lambda_{2}$ be the inclusion of $T_{2}$ in $\mathcal{A} \otimes T_{2}$. Then letting $\tau_{i}=\tau \circ \lambda_{i}$ for $i=1,2$, we have $\tau_{1} \odot \tau_{2}=\tau$.

Since $\mathcal{A}$ and $T_{2}$ have interpolating spectrum, the locally contractive representations $\tau_{1}$ and $\tau_{2}$ are completely contractive. Thus we have a representation of $\mathcal{A} \otimes T_{2}$ of the form $\tau_{1} \odot \tau_{2}$ that has no $*$-dilation. By Paulsen and Power's result mentioned above, it follows that $\mathcal{A}$ does not have the commutant lifting property.

\section{References}

[An] T. Ando, On a pair of commuting contractions, Acta Sci. Math. (Szeged), 24 (1963), 88-90. 
[A1] W.B. Arveson, Subalgebras of $\mathrm{C}^{*}$-algebras, Acta Math., 123 (1969), 141-224.

[A2] _ Subalgebras of $\mathrm{C}^{*}$-algebras, II, Acta Math., 128 (1972), 271-308.

[D] K.R. Davidson, When locally contractive representations are completely contractive, J. Funct. Anal., 128 (1995), 186-225.

[DPP] K.R. Davidson, V.I. Paulsen, and S.C. Power, Tree algebras, semidiscreteness and dilation theory, Proc. London Math. Soc., 68(3) (1994), 178-202.

[DP] K.R. Davidson and S.C. Power, Isometric automorphisms and homology for nonself-adjoint operator algebras, Quart. J. Math. Oxford Ser., 42(2) (1991), 271-292.

[DoH] A.P. Donsig and A. Hopenwasser, Order preservation in limit algebras, J. Funct. Anal., 133 (1995), 342-394.

[DoP] A.P. Donsig and S.C. Power, The failure of approximate inner conjugacy for standard diagonals in regular limit algebras, Canad. Math. Bull., 39 (1996) 375-398.

[ER] E.G. Effros and Z.J. Ruan, On non-self adjoint operator algebras, Proc. Amer. Math. Soc., 110 (1990), 915-922.

[GHL] F. Gilfeather, A. Hopenwasser and D.R. Larson, Reflexive algebras with finite width lattices: Tensor products, cohomology, compact perturbations, J. Funct. Anal., 55 (1984), 175-198.

[HP] A. Hopenwasser and S.C. Power, Classification of limits of triangular matrix algebras, Proc. Edinburgh Math. Soc., 36 (1992), 107-121.

[MM] M. McAsey and P.S. Muhly, Representations of non-self-adjoint crossed products, Proc. London Math. Soc., 47(3) (1983), 128-144.

[MS1] P.S. Muhly and B. Solel, Dilations for representations of triangular algebras, Bull. London Math. Soc., 21 (1989), 489-495.

[MS2] Subalgebras of groupoid C $\mathrm{C}^{*}$-algebras, J. Reine Angew. Math., 402 (1989), 41-75.

[MS3] , Dilations and commutant lifting for subalgebras of groupoid $\mathrm{C}^{*}$-algebras, Internat. J. Math., 5 (1994), 87-123.

[MS4] Hilbert modules over operator algebras, Mem. Amer. Math. Soc., no. 559, Amer. Math. Soc., Providence, RI, 1995.

[Pa] S. Parrott, Unitary dilations for commuting contractions, Pacific J. Math., 34 (1970), 481-490.

[PP1] V.I. Paulsen and S.C. Power, Lifting theorems for nest algebras, J. Operator Theory, 20 (1987), 312-327.

[PP2] , Tensor products of non-self-adjoint operator algebras, Rocky Mountain J. Math., 20 (1990), 331-350.

[PPS] V.I. Paulsen, S.C. Power and R.R. Smith, Schur products and matrix completions, J. Funct. Anal., 85 (1989), 151-178.

[PPW] V.I. Paulsen, S.C. Power and J. Ward, Semidiscreteness and dilation theory for nest algebras, J. Funct. Anal., 80 (1988), 76-87.

[P1] S.C. Power, The classification of triangular subalgebras of AF $\mathrm{C}^{*}$-algebras, Bull. London Math. Soc., 22 (1990), 269-272.

[P2] , Non-self-adjoint operator algebras and inverse systems of simplicial complexes, J. Reine Angew. Math., 421 (1991), 43-61. 
[P3] Limit algebras, Pitman Research Notes in Mathematics, no. 278, Longman Scientific and Technical, London, 1992.

[SN] B. Sz.-Nagy, Sur les contractions de l'espace de Hilbert, Acta Sci. Math. (Szeged), 15 (1953), 89-92.

[SNF1] B. Sz.-Nagy and C. Foiaş, Dilatations des commutants d'opérateurs, C.R. Acad. Sci. Paris, Sér. A, 266 (1968), 493-495.

[SNF2] Harmonic analysis of operators on Hilbert space, North-Holland Publishing Co., Amsterdam \& London, 1970, Translated from the French and revised.

[T] M. Thelwall, Dilation theory for subalgebras of AF algebras, J. Operator Theory, 25 (1991), 275-282.

Received August 10, 1996 and revised August 20, 1997. The author was partially supported by an NSERC of Canada Postdoctoral Fellowship.

UNIVERSITY OF WATERLOO

Waterloo, Canada, N2L 3G1

Current address:

UNIVERSITY OF NEBRASKA-LINCOLN

LINCOLN, NE 68588-0323

E-mail address: adonsig@math.unl.edu 